$\Rightarrow$ RENAL INJURY

\section{Intu protects the kidney}

The establishment and maintenance of planar cell polarity (PCP) has an essential role in embryonic development and in normal tissue function. New findings from Zheng Dong and colleagues suggest that the PCP effector protein inturned (Intu) also protects against renal ischaemia-reperfusion injury (IRI) by promoting cell survival and ciliogenesis.

Several PCP effector proteins, including Intu, have roles in the regulation of ciliogenesis, so are classified as ciliogenesis and planar polarity effector proteins. "Previous work from our group and others has implicated primary cilia in kidney injury," comments Dong. "However, the role of PCP proteins in kidney injury and repair is poorly understood."

To investigate this role, the researchers generated mice with specific knockout of Intu in the proximal tubules. They report that renal IRI induced Intu expression in the kidneys of wild-type mice but not in those of the Intu knockouts. Following renal IRI, renal tubule damage and cell death were increased and post-injury ciliogenesis was reduced in the Intu-knockout mice compared with wild-type controls. "These findings provide the first compelling evidence for an involvement of PCP in the response to renal IRI," says Dong.

Using tandem affinity purification in conjunction with mass spectrometry, the researchers identified signal transducer and activator of transcription 1 (STAT1) as a novel interacting partner of Intu. In proximal tubule cells, Intu and STAT1 colocalized at the centriole and basal body area. During cell stress, Intu expression suppressed STAT1 expression, reduced cell death and preserved cilia length, whereas Intu deficiency resulted in accumulation of STAT1 and increased cell death and ciliary defects. Knockdown of STAT1 attenuated these effects in Intu-silenced cells.

"Mechanistically, we have identified a novel interaction between Intu and STAT1," says Dong. "Our findings suggest that by interacting with STAT1, Intu may promote STAT1 degradation via the proteasome, resulting in ciliogenesis and cell survival during cell stress. Together, these results indicate that Intu protects cells and tissues from injury by targeting STAT1 for degradation and maintaining primary cilia."

Ellen F. Carney

ORIGINAL ARTICLE Wang, S. et al. The CPLANE protein Intu protects kidneys from ischemia-reperfusion injury by targeting STAT1 for degradation. Nat. Commun. 9, 1234 (2018)

\title{
A neutrophil motility assay for early diagnosis of sepsis
}

Current diagnostic methods for sepsis that are in clinical use lack precision, often leading to misdiagnoses. Although microbiological cultures can aid diagnosis, they usually require 2-3 days for bacterial growth and are therefore unsuitable for early diagnosis and treatment. Now, Daniel Irimia and colleagues report that patients with sepsis can be distinguished from those without sepsis based on differential neutrophil motility patterns inside microfluidic devices.

The researchers previously observed a sepsis-specific spontaneous motility signature of isolated neutrophils. They have now developed an assay in which neutrophils from a diluted whole-blood sample autonomously pass through a filter that largely prevents other cells from entering the migration channels. This approach enables the assessment of neutrophil motility in their physiological and biochemical environment, without the need for isolation procedures and chemoattractant gradients. Five motility parameters (neutrophil count, average distance travelled and the frequency of oscillation, pausing and reverse migration events) were identified as characteristic for sepsis using a machine learning-based approach. These parameters were used to develop a scoring system that stratified critically ill patients suspected of sepsis with $98 \%$ specificity and $97 \%$ sensitivity.

"Our findings suggest that neutrophils have an important role in the pathology of sepsis, which could be exploited using interventions that target neutrophils in addition to current antimicrobial and supportive therapies," says Irimia.

Next, the researchers plan to investigate the underlying molecular mechanisms of spontaneous neutrophil motility in sepsis, as well as to validate and refine the sepsis score and threshold in a larger and more diverse cohort of at-risk patients.

Jack M. Heintze

ORIGINAL ARTICLE Ellett, F. et al. Diagnosis of sepsis from a drop of blood by measurement of spontaneous neutrophil motility in a microfluidic assay. Nat. Biomed. Eng. https://doi. org/10.1038/s41551-018-0208-z (2018)

\section{GLOMERULAR DISEASE}

\section{Osteopontin drives renal metabolic dysfunction in Alport syndrome}

New data from Lina Shehadeh and colleagues suggest that osteopontin is a potential therapeutic target for Alport syndrome. Increased expression of this phosphoprotein has previously been reported in various diseases, including nephrotic syndrome. "At basal levels, osteopontin is mainly expressed in the bones and to some extent in the kidneys and gall bladder," says Shehadeh. "However, when there is stress or inflammation, osteopontin expression becomes exponentially high in the diseased organ and in the circulation."

To investigate the role of osteopontin in Alport syndrome, the researchers used the $\mathrm{Col}_{4} \mathrm{a}^{-1-}$ mouse model of this disease. They report increased expression of osteopontin, dynamin 3 and the low-density lipoprotein receptor (LDLR) as well as cholesterol accumulation and dysmorphic mitochondria in the kidney tubules of these mice compared with wild-type controls. Heterozygous deletion of osteopontin increased lifespan and reduced renal, ocular and cochlear pathologies, cholesterol accumulation and the expression of dynamin 3 and LDLR in Col4a $3^{-/-}$mice. Further investigations using human renal epithelial cells demonstrated that osteopontin induces the expression of dynamin 3, which in turn upregulates LDLR, resulting in increased cholesterol influx. Dynamin 3 overexpression also resulted in impaired mitochondrial respiration in renal epithelial cells, suggesting a causative role of increased dynamin 3 levels in mitochondrial dysfunction in Alport syndrome.

"Our findings identify a new pathway that may be responsible for renal cholesterol accumulation and metabolic dysfunction in Alport syndrome," concludes Shehadeh. "Importantly, the osteopontin-dynamin 3-LDLR pathway may also have a role in other degenerative diseases in which cholesterol and/or lipid toxicity are prominent, such as atherosclerosis and cancer."

Ellen F. Carney

ORIGINAL ARTICLE Ding, W. et al. Osteopontin deficiency ameliorates Alport pathology by preventing tubular metabolic deficits. JCI lnsight 3, e94818 (2018) 